\title{
Observation of Broadband Entanglement in Microwave Radiation from a Single Time-Varying Boundary Condition
}

\author{
B. H. Schneider®, A. Bengtsson, I. M. Svensson, T. Aref, G. Johansson, Jonas Bylander, and P. Delsing \\ Department of Microtechnology and Nanoscience (MC2), Chalmers University of Technology, SE-412 96 Gothenburg, Sweden
}

(Received 22 September 2018; revised manuscript received 28 February 2020; accepted 27 March 2020; published 10 April 2020)

\begin{abstract}
Entangled pairs of microwave photons are commonly produced in the narrow frequency band of a resonator, which represents a modified vacuum density of states. We generate and investigate the entanglement of a stream of photon pairs, generated in a semi-infinite broadband transmission line, terminated by a superconducting quantum interference device (SQUID). A weak pump signal modulates the SQUID inductance, resulting in a single time-varying boundary condition, and we detect all four quadratures of the microwave radiation emitted at two different frequencies separated by $0.7 \mathrm{GHz}$. Power calibration is done in situ, and we find positive logarithmic negativity and two-mode squeezing below the vacuum in the observed radiation, indicating entanglement.
\end{abstract}

DOI: 10.1103/PhysRevLett.124.140503

A time-varying boundary condition for the electromagnetic field can generate entangled photon pairs from the quantum vacuum. This fundamental property is called the dynamical Casimir effect (DCE) [1]. At low temperatures, the resulting output radiation due to the DCE exhibits twomode squeezing [2], which means that a portion of the noise is shared between two modes. Whenever the ratio of the shared noise to the nonshared noise in two detected modes is larger than a certain threshold [3], the two modes are quantum entangled. Quantum backaction, e.g., due to a projective measurement of one mode, then affects both modes.

Sources of entangled optical photons have been used in secure quantum key distribution [4], quantum repeaters [5], and quantum sensing applications [6]. At microwave frequencies, two-mode entanglement was proposed for entangling qubits [7], for continuous-variable quantum computing [8] and quantum enhanced detection at ambient conditions $[9,10]$. Sources of microwave entanglement such as parametric amplifiers [11-13] and modulated nonlinear media [14] are comprised of a time-varying boundary condition or light velocity within a cavity; this arrangement enhances the radiation within the relatively narrow bandwidth of the cavity but suppresses it outside of this band. In contrast, broadband entanglement sources are not as common [15] but are useful for two reasons: (i) they can be very bright and generate a large number of entangled photons and (ii) their wide frequency content allows for the

Published by the American Physical Society under the terms of the Creative Commons Attribution 4.0 International license. Further distribution of this work must maintain attribution to the author(s) and the published article's title, journal citation, and DOI. Funded by Bibsam. shaping of the emitted radiation in time. Good temporal control over the photon generation process is required in order to shape photon packages [16,17]. Protocols that reach unity efficiency in transmitting and absorbing photons rely on such temporal shaping [18-21].

Broadband two-mode squeezing of microwave radiation was demonstrated by means of the DCE in a transmission line [2]. However, to demonstrate entanglement, these correlations should be compared with the correlations within each mode, where the amplifier noise needs to be subtracted. In the previous setup, imprecision in the determination of the output photon flux and a nonlinearity due to strong pumping precluded the unequivocal demonstration of entanglement between photons. Since then, clear quantitative bounds for entanglement have been derived [22], taking thermal photons into account.

In this Letter we demonstrate broadband entanglement of microwave photon pairs generated by the DCE in a superconducting circuit. The circuit consists of a semiinfinite transmission line, terminated by two parallel Josephson junctions connected to ground (a direct current superconducting quantum interference device, dc SQUID); see Fig. 1. This SQUID's Josephson inductance represents a variable boundary condition that mimics a "movable mirror" for the electromagnetic field [23]. We rapidly modulate this boundary condition, at a microwave frequency $\left(f_{p}=8.9 \mathrm{GHz}\right)$, by means of pumping the magnetic flux threading the SQUID loop, thereby producing DCE radiation.

We detect the in-phase and quadrature voltages of the output field of the transmission line at different pump amplitudes, i.e., at different displacement speeds of the electromagnetic boundary condition. We then compute the covariance matrix of the voltage fluctuations at two different frequencies $f_{+}$and $f_{-}$(where $f_{+}+f_{-}=f_{p}$ and 
$f_{+}-f_{-}=0.7 \mathrm{GHz}$ ), and further determine the lognegativity and calculate the amount of two-mode squeezing below the vacuum level. This quantification of entanglement relies on our careful calibration of the system gain and noise level and the flux-pump amplitude [24]. Measurements were done in a dilution refrigerator at a temperature of $10 \mathrm{mK}$. The device under test consists of an aluminum dc SQUID with a loop area of $6 \times 8 \mu \mathrm{m}^{2}$, directly connected to a $0.6 \mathrm{~mm}$ long on-chip superconducting niobium transmission line. The ground plane and fluxpump antenna are also made in the same niobium layer. A semiconductor HEMT (high electron mobility transistor) amplifier with $39 \mathrm{~dB}$ gain amplifies the signals in the range of 4-8 GHz. After additional amplification and filtering, two digitizers detect the heterodyne down-converted signals at two frequencies, yielding the quadrature voltages $I$ and $Q$ at frequencies $f_{+}=4.8 \mathrm{GHz}$ and $f_{-}=4.1 \mathrm{GHz}$. The digitizer bandwidth is set to $100 \mathrm{kHz}$ and each data point presented is averaged for 100 seconds resulting in ten million voltage values for the $I$ and $Q$ quadratures each.

A probe signal can be launched via the circulator and is used to characterize the change in phase and magnitude of the reflected signal. Furthermore, four low-pass-filtered wires are connected across the SQUID to enable the dc characterization. The low-pass filtering in these lines consists of high resistance-capacitance and copper-powder filters with a total cutoff frequency of $30 \mathrm{~Hz}$. An external magnetic coil is used to set the static flux $\left(\Phi_{\mathrm{dc}}\right)$ of the SQUID. We modulate the boundary condition by sending an ac signal $\left(\Phi_{\mathrm{ac}}\right)$ to an on-chip flux pump line [Fig. 1(b)]. The flux-pumping frequency can be chosen arbitrarily.

We first characterize the device by sourcing a current and measuring the voltage across the SQUID [Fig. 2(a)] and find a critical current of $I_{c}=3.4 \mu \mathrm{A}$ and a superconducting energy gap voltage of $360 \mu \mathrm{eV}$. From the forward (blue) and backwards (red) current sweeps, we observe a hysteresis, indicating that the SQUID is underdamped, with $\beta_{C} \approx$ $\left[4 I_{c} /\left(\pi I_{r}\right)\right]^{2} \approx 10^{4}[25,26]$, where $I_{r} \approx 45 \mathrm{nA}$ is the superconducting retrapping current.

To obtain the necessary resolution in the measurement of the voltage output from the transmission line, we use the SQUID itself to calibrate the noise and gain of the amplifier chain [Fig. 2(b)]. By applying a current through the SQUID, shot noise is generated [27], which can be used to calibrate the system $[28,29]$. At the same time, the voltage drop across the SQUID is measured, determining its resistance. The resistance of the SQUID for a voltage above the gap, $V_{g}=(2 \Delta / e)$, is $R=69.7 \Omega$ [Fig. 2(a)]. The difference compared to the impedance of the transmission line, $Z_{0}=50 \Omega$, is taken into account using the following equations:

$$
\begin{aligned}
& S_{p}=G B w\left[\frac{V_{T}^{2}}{2 Z_{0}}\left(\frac{E_{1}}{\tanh \left(E_{1}\right)}+\frac{E_{2}}{\tanh \left(E_{2}\right)}\right)+k_{B} T_{n}\right], \\
& E_{1}=\frac{V_{s}^{2}+V_{z}^{2}}{V_{T}^{2}}, \quad E_{2}=\frac{V_{s}^{2}-V_{z}^{2}}{V_{T}^{2}},
\end{aligned}
$$

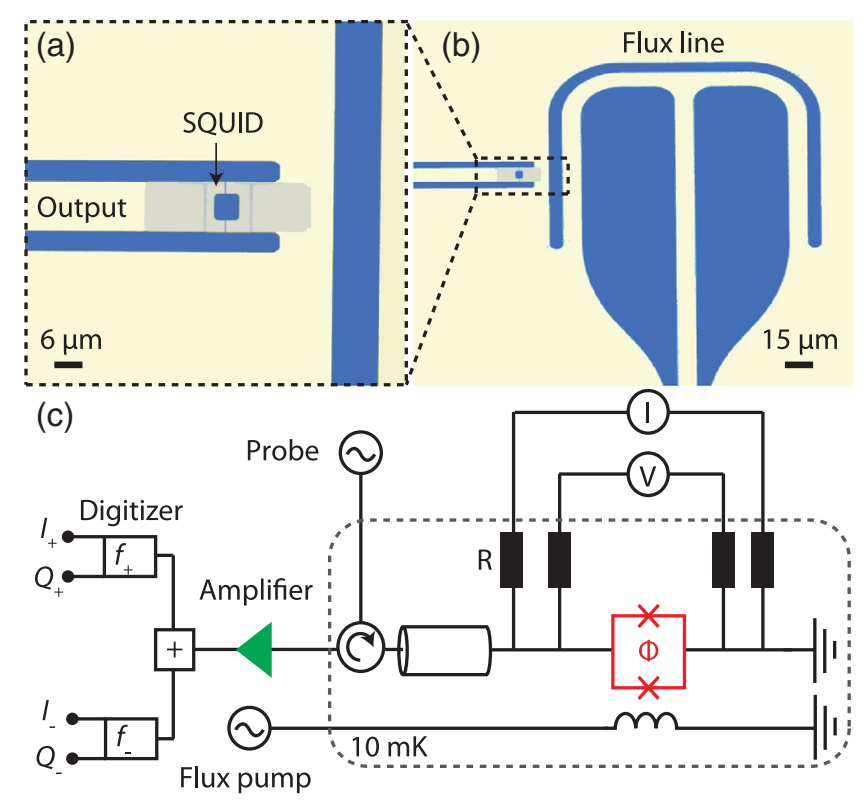

FIG. 1. False-color photograph and simplified circuit schematic of the measured sample. (a),(b) The dc SQUID is made of aluminum (grey) and connects a coplanar waveguide transmission line to ground. The transmission line and the ground plane are made of niobium (yellow) on top of a sapphire substrate (blue). (b) On-chip magnetic flux line located next to the SQUID. (c) Simplified circuit diagram showing cables carrying alternating and direct currents to the device.

where $k_{\mathrm{B}}$ is Boltzmann's constant, $T$ is the device temperature, $T_{n}$ is the system noise temperature referred to the device, and $G$ and $B w$ are the gain and the detection bandwidth, respectively. $V_{s}^{2}=2 e|I| R^{2} \cdot Z_{0}^{2} /\left(Z_{0}+R\right)^{2}$, $V_{T}^{2}=4 k_{B} T \cdot Z_{0}^{2} /\left(Z_{0}+R\right)$ and $V_{z}^{2}=Z_{0} \cdot \frac{1}{2} h f$ are spectral densities, which relate to the shot noise, Johnson noise, and zero-point fluctuations at frequency $f$, respectively. $h$ is Planck's constant. $S_{p}$ is the integrated shot noise power spectral density measured within the bandwidth $B w$.

In Fig. 2(b), we show the spectral density of the shot noise and the corresponding fit as a function of dc current through the SQUID, with a static magnetic flux of $\Phi_{\mathrm{dc}}=-0.47 \Phi_{0}$, which is used throughout the paper. Here $\Phi_{0}=h / 2 e$ is the magnetic flux quantum. This fit accurately determines the system noise temperature and gain. The system noise corresponds to a temperature of $3.71 \pm 0.04 \mathrm{~K}$ at $4.1 \mathrm{GHz}$ and $2.95 \pm 0.02 \mathrm{~K}$ at $4.8 \mathrm{GHz}$, which matches the noise of the HEMT amplifier which is $2.3 \mathrm{~K}$ at $4.1 \mathrm{GHz}$ and $2 \mathrm{~K}$ at $4.8 \mathrm{GHz}$, connected via two circulators and filters. Since the HEMT amplifier dominates the noise, we can determine the corresponding photon losses between device and amplifier. Here we find a photon $\operatorname{loss}$ of $10 \log (2.2 / 3.7) \approx-2.3 \mathrm{~dB}$ at $4.1 \mathrm{GHz}$ and $10 \log (2 / 2.95) \approx-1.7 \mathrm{~dB}$ at $4.8 \mathrm{GHz}$.

To generate DCE photons, we apply a sinusoidal signal to the flux line at $f_{p}=f_{-}+f_{+}=8.9 \mathrm{GHz}$, while recording the signal using two digitizers at $f_{-}=4.1 \mathrm{GHz}$ and 

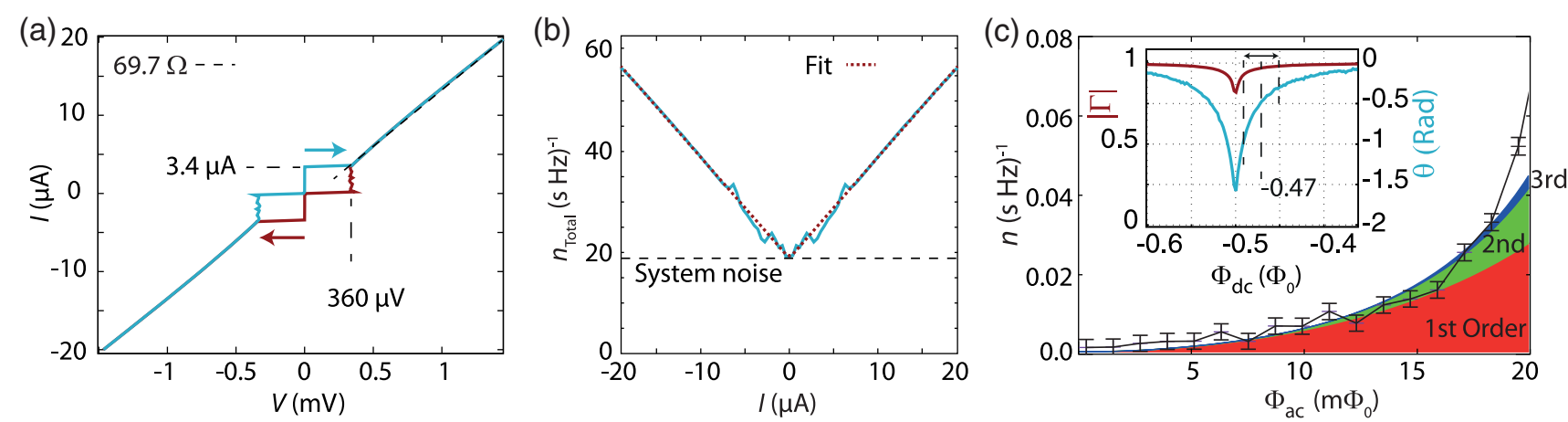

FIG. 2. SQUID characterization and circuit calibration. (a) Current-voltage characteristic of the SQUID (up-sweep blue, down-sweep red). The high-bias resistance is $69.7 \Omega$ (dashed line). (b) Shot noise photon spectral density at $4.1 \mathrm{GHz}$ versus applied current. (c) Average photon spectral density generated as a function of flux-pump amplitude. A flux modulation of $20 \mathrm{~m} \Phi_{0}$ at $f_{p}$ corresponds to an equivalent mirror speed of $31 \%$ of the speed of light. The inset shows the magnitude $|\Gamma|$ (red) and the relative phase change $\theta$ (blue) of the reflected probe signal at $4.1 \mathrm{GHz}$. A vertical dashed line at $-0.47 \Phi_{\mathrm{dc}}$ indicates the static flux position used and the effective flux modulation range for $20 \mathrm{~m} \Phi_{0}$ is also indicated. A model is used to fit the resulting photon rate taking the first three orders of the SQUID nonlinearity into account. The effective photon number contributions for the individual modes are color coded and marked in the figure.

$f_{+}=4.8 \mathrm{GHz}$, i.e., placed symmetrically around $f_{p} / 2$ [Fig. 1(c)]. DCE photons are generated in pairs symmetrically around half the pump frequency. By using a photon spectral density we can compare the photon rates. The effective speed of "mirror" displacement is given by the phase response [inset of Fig. 2(c)], the flux amplitude $\Phi_{\mathrm{ac}}$, and $f_{p}$, thus $v_{\text {eff }}=f_{p} \Phi_{\mathrm{ac}} \cdot \delta \theta / \delta \Phi_{\mathrm{dc}} \cdot\left(2 c / f_{p}\right)$, where $\delta \theta / \delta \Phi_{\mathrm{dc}}$ is the phase change with magnetic field and $2 c / f_{p}$ is the wavelength at half the pump frequency. For small amplitudes, the phase depends linearly on the flux, such that the boundary condition can be mapped to a sinusoidally moving mirror. With a flux pump amplitude $\Phi_{\text {ac }}$ exceeding $15 \mathrm{~m} \Phi_{0}$, the change in phase becomes larger, which results in a larger photon spectral density; however, the motion also becomes nonlinear [24]. A power calibration of the flux pump amplitude $\Phi_{\mathrm{ac}}$ is described in the Supplemental Material [24].

We experimentally track changes in the output radiation such as photons generated by the DCE. We do this by switching the pump on and off and tracking the difference. From the calibration [Figs. 2(a) and 2(b)], we obtained a photon spectral density of $0.5 \pm 0.0035(\mathrm{~s} \mathrm{~Hz})^{-1}$ corresponding to the vacuum fluctuations, when the pump is switched off, and the system noise is subtracted. The background noise in the system is determined by subtracting the amplifier noise and the zero-point fluctuations from the total detected noise. Any remaining noise signal are due to thermal photons. This is smaller than what we could resolve, confirming a photon temperature of less than $40 \mathrm{mK}$. However, the uncertainty in the background noise $\pm 0.0035(\mathrm{~s} \mathrm{~Hz})^{-1}$ is not small enough to resolve temperature below $40 \mathrm{mK}$, which corresponds to $n_{t h}=$ $1 /\left\{\exp \left[h f /\left(k_{B} T\right)\right]-1\right\}=0.0031$ at $4.8 \mathrm{GHz}$. As we increase the flux pump amplitude, we measure an increase in photon spectral density. Figure 2(c) shows the generated photon spectral density versus flux pump amplitude.

As described in detail in [24] we model the sample as a tunable boundary condition to the electromagnetic field in the transmission line. For a sinusoidal modulation of the boundary condition, the DCE photon rate has a parabolic spectrum centered around $f_{p} / 2$. If the phase-flux dependence is nonlinear, as is the case here, the boundary condition is effectively modulated not only at the pump frequency $f_{p}$, but also at integer multiples, $n f_{p}$. This modifies the DCE photon rate: each harmonic will give rise to a parabolic spectrum centered around $n f_{p} / 2$. By taylor expanding the measured phase as a function of flux, we can predict the photon rate contributions from each parabola. This prediction, with no fitting parameters, is plotted in Fig. 2(c) together with the measured data. We find that this model describes the photon rate well.

We use two methods to probe and characterize entanglement between produced photon pairs: first, by calculating the log-negativity, and, second, by comparing the quadrature noise to that of the vacuum. Both methods are commonly used to probe entanglement and nonseparability [30,31].

As we generate photons using the DCE, shown in Fig. 2(c), we record the voltage quadratures $I_{+}, Q_{+}, I_{-}$, and $Q_{-}$corresponding to the frequencies $f_{+}$and $f_{-}$. From the quadrature correlations, we can construct the covariance matrix [inset in Fig. 3(a)]. The inset in Fig. 3(b) shows the covariance matrix with subtracted contributions from the zero-point fluctuations. Error values are estimated for all elements of the covariance matrix as one standard deviation. Once the covariance matrix is established, we calculate the logarithmic negativity [22]:

$$
\begin{aligned}
\mathcal{N} & =\max \left[0,-\log _{2}\left(2 \nu_{-}\right)\right], \\
\nu_{-} & =\left[\zeta / 2-\left(\zeta^{2}-4 \operatorname{det} V\right)^{1 / 2} / 2\right]^{1 / 2}, \\
\zeta & =\operatorname{det} A+\operatorname{det} B-2 \operatorname{det} C, \\
V & =\frac{1}{2}\left(\begin{array}{cc}
A & C \\
C^{T} & B
\end{array}\right),
\end{aligned}
$$



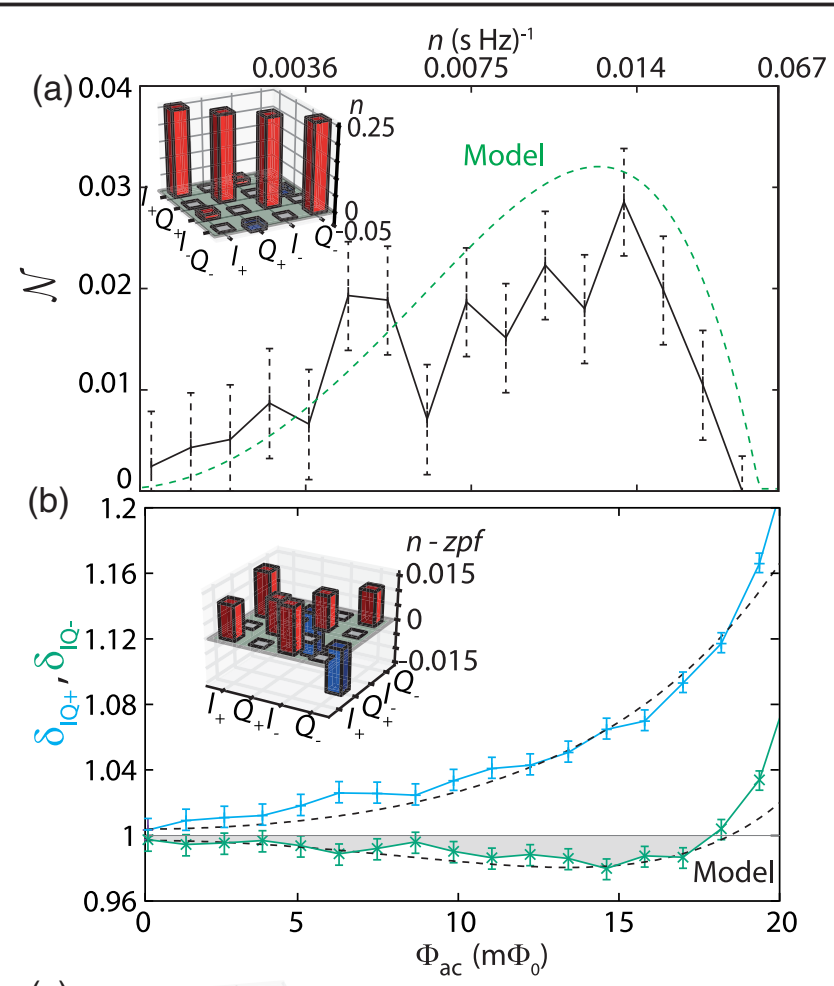

(c)

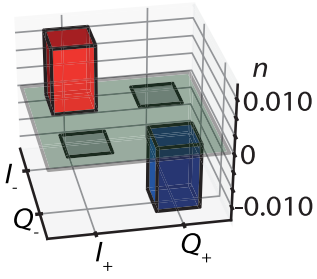

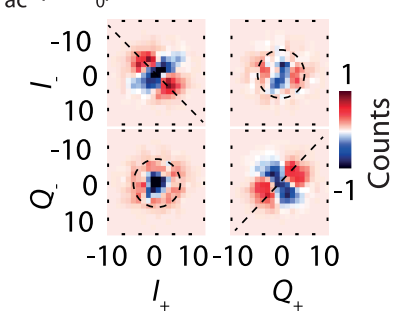

FIG. 3. Two measures of entanglement (a),(b) as a function of flux pump amplitude $\Phi_{\mathrm{ac}}$ and photon spectral density $n$. The results of the model are plotted on top (dashed lines). (a) Logarithmic negativity $\mathcal{N}>0$ indicates entanglement. The inset shows a covariance matrix, taken at a photon spectral density of $n=0.01$ and flux pump amplitude of $\Phi_{\text {ac }}=12.5 \mathrm{~m} \Phi_{0}$. (b) combined quadrature fluctuations in the $I$ and $Q$ quadratures. At low amplitudes, we observe squeezing below the vacuum in both quadratures (shaded grey) when $\delta_{I Q-}<1$, fulfilling the inseparability criterion [3]. The inset shows the same covariance matrix as the inset in (a), however, with the zero-point fluctuations at zero driving subtracted. (c) The two insets show the off-diagonal elements of the covariance matrix with the corresponding quadrature histograms. Each histogram is calculated from the difference between flux pump on and off.

where $V$ is the $4 \times 4$ covariance matrix with the $2 \times 2$ submatrices $A, B$, and $C$. The logarithmic negativity is positive for flux pump amplitudes below $18 \mathrm{~m} \Phi_{\mathrm{ac}}$ [Fig. 3(a)], and follows the results of the model. For $\Phi_{\mathrm{ac}}$ larger than $\sim 10 \mathrm{~m}_{0}$, the second parabola becomes significant. Since photons from this parabola are not entangled at the two measurement frequencies, the added photons appears thermal, and the log-negativity will therefore start to decrease. This is captured well by the model [24] shown as a dashed line in Fig. 3(c). The logarithmic negativity is lower than the theoretical ideal value [22] $(\mathcal{N} \approx 2 \sqrt{n}=0.2)$. This is because the equation neglects anything related to non-linearities, losses and noise. Similarly to Ref. [32], we include measurement noises and losses in the presented results. Photon losses in the system and the nonlinearity [24] in the SQUID result in lower cross-correlation values. Taking these effects into account in the model, we obtain values close to the measured results.

The right part of Fig. 3(c) shows four histograms of measured $I$ and $Q$ quadratures, taken at a flux pump amplitude $\Phi_{\mathrm{ac}}=13 \mathrm{~m} \Phi_{0}$. The histograms show the difference between flux pump on and off. The top left $I_{-} I_{+}$ histogram and bottom right $Q_{-} Q_{+}$histogram show squeezing along the dashed diagonals that are orthogonal to each other: the radiation is amplified along the diagonal dashed line and is squeezed orthogonally to it. The left part of Fig. 3(c) shows the corresponding subset of the covariance matrix.

From the quadrature correlations, we calculate the combined quadrature fluctuations $\delta_{I Q+}=\left\langle\left(I_{+}+I_{-}\right)^{2}\right\rangle+$ $\left\langle\left(Q_{+}-Q_{-}\right)^{2}\right\rangle$ and $\delta_{I Q_{-}}=\left\langle\left(I_{+}-I_{-}\right)^{2}\right\rangle+\left\langle\left(Q_{+}+Q_{-}\right)^{2}\right\rangle$ as a function of flux pump amplitude [Fig. 3(b)], where the later fulfils the inseparability criterion for continuous variable systems by Duan $[3,33]$ for values below 1 . We observed $-0.09 \pm 0.02 \mathrm{~dB}$ squeezing below the vacuum in $\delta_{I Q-}$. We also observed an amplification of $0.25 \pm 0.02 \mathrm{~dB}$ in $\delta_{I Q+}$ at a flux pump strength of $\Phi_{\mathrm{ac}}=15 \mathrm{~m} \Phi_{0}$. Again this behaviour is captured by our model which is shown as dashed lines in Fig. 3(b). For low flux pump powers in the more linear regime, both methods indicate entanglement. The two modes for which we find entanglement are part of a larger spectrum of photons generate by the DCE; thus, we conclude that the DCE does produce entanglement as theoretically predicted.

To compare the entanglement generation, we calculate the rate of entangled bits with the entropy of formation [34] for a given logarithmic negativity of 0.03 , which is $E_{F}=$ $(1.6 \pm 0.3) \times 10^{-3}$ at the amplifier input [24]. This corresponds to an entanglement rate of $\sim 5.2 \mathrm{Mebit} / \mathrm{s}$, in turn corresponding to a distribution rate of entangled Bell pairs [35]. These numbers are substantially larger at the device. There are two reasons for this: losses between the device and the amplifier and the limited bandwidth of the amplifier. Taking losses into account and a bandwidth from 3.2 to $5.7 \mathrm{GHz}$ (to exclude thermal photons), we estimate $E_{F}=13 \times 10^{-3}$ available at the device, corresponding to an entanglement rate of $\sim 30 \mathrm{Mebit} / \mathrm{s}$. This is similar to [15] and high in comparison to cavity-based entanglement sources [24].

To improve the entanglement rate of this device, the following changes are suggested: a higher plasma frequency of the SQUID would allow driving at higher frequencies, thus increasing the output bandwidth and photon production rate. This can be done by using a 
thinner oxide layer for the junctions. The quality of generated DCE photons can be improved by placing a bandpass filter with a feature size smaller than one tenth of the output wavelength in front of the device. This would tailer the vacuum modes outside the pass band in such a way that nonlinear components are suppressed. We note that it should also be possible to modulate the boundary condition in a nonsinusodial way to cancel the higher order parabolas. This should allow for more purely entangled photons and a higher entanglement rate.

In conclusion, we demonstrated that photon pairs generated by the DCE without a cavity are entangled. To our knowledge, entanglement of a single time-varying boundary condition without the presence of a cavity has previously not been observed.

We thank C. M. Wilson, W. Wieczorek, V. Shumeiko, and N. Treps for useful discussions on methods and entanglement. We gratefully acknowledge financial support from the European Research Council, the European project PROMISCE, the Swedish Research Council, and the Wallenberg Foundation. J. B. acknowledges partial support by the EU under REA Grant Agreement No. CIG-618353.

[1] G. T. Moore, J. Math. Phys. (N.Y.) 11, 2679 (1970).

[2] C. M. Wilson, G. Johansson, A. Pourkabirian, M. Simoen, J. R. Johansson, T. Duty, F. Nori, and P. Delsing, Nature (London) 479, 376 (2011).

[3] L.-M. Duan, G. Giedke, J. I. Cirac, and P. Zoller, Phys. Rev. Lett. 84, 2722 (2000)

[4] A. K. Ekert, Phys. Rev. Lett. 67, 661 (1991).

[5] H.-J. Briegel, W. Dür, J. I. Cirac, and P. Zoller, Phys. Rev. Lett. 81, 5932 (1998).

[6] P. B. Dixon, G. A. Howland, Kam Wai Clifford Chan, C. O’Sullivan-Hale, B. Rodenburg, N. D. Hardy, J. H. Shapiro, D. S. Simon, A. V. Sergienko, R. W. Boyd, and J. C. Howell, Phys. Rev. A 83, 051803(R) (2011).

[7] S. Felicetti, M. Sanz, L. Lamata, G. Romero, G. Johansson, P. Delsing, and E. Solano, Phys. Rev. Lett. 113, 093602 (2014).

[8] U. L. Andersen, J. S. Neergaard-Nielsen, P. van Loock, and A. Furusawa, Nat. Phys. 11, 713 (2015).

[9] S. Barzanjeh, S. Guha, C. Weedbrook, D. Vitali, J. H. Shapiro, and S. Pirandola, Phys. Rev. Lett. 114, 080503 (2015).

[10] S. Barzanjeh, E. S. Redchenko, M. Peruzzo, M. Wulf, D. P. Lewis, G. Arnold, and J. M. Fink, Nature (London) 570, 480 (2019).
[11] C. Eichler, D. Bozyigit, C. Lang, M. Baur, L. Steffen, J. M. Fink, S. Filipp, and A. Wallraff, Phys. Rev. Lett. 107, 113601 (2011).

[12] L. Zhong, E. P. Menzel, R. Di Candia, P. Eder, M. Ihmig, A. Baust, M. Haeberlein, E. Hoffmann, K. Inomata, T. Yamamoto, Y. Nakamura, E. Solano, F. Deppe, A. Marx, and R. Gross, New J. Phys. 15, 125013 (2013).

[13] C. W. Sandbo Chang, M. Simoen, J. Aumentado, C. Sabín, P. Forn-Díaz, A. M. Vadiraj, F. Quijandría, G. Johansson, I. Fuentes, and C. M. Wilson, Phys. Rev. Applied 10, 044019 (2018).

[14] P. Lähteenmäki, G. S. Paraoanu, J. Hassel, and P. J. Hakonen, Proc. Natl. Acad. Sci. U.S.A. 110, 4234 (2013).

[15] J.-C. Forgues, C. Lupien, and B. Reulet, Phys. Rev. Lett. 114, 130403 (2015).

[16] A. L. C. Rego, H. O. Silva, D. T. Alves, and C. Farina, Phys. Rev. D 90, 025003 (2014).

[17] H. O. Silva and C. Farina, Phys. Rev. D 84, 045003 (2011).

[18] J. I. Cirac, P. Zoller, H. J. Kimble, and H. Mabuchi, Phys. Rev. Lett. 78, 3221 (1997).

[19] K. Jahne, B. Yurke, and U. Gavish, Phys. Rev. A 75, 010301 (R) (2007).

[20] A. N. Korotkov, Phys. Rev. B 84, 014510 (2011).

[21] M. Pechal, L. Huthmacher, C. Eichler, S. Zeytinoğlu, A. A. Abdumalikov, S. Berger, A. Wallraff, and S. Filipp, Phys. Rev. X 4, 041010 (2014).

[22] J. R. Johansson, G. Johansson, C. M. Wilson, P. Delsing, and F. Nori, Phys. Rev. A 87, 043804 (2013).

[23] J. R. Johansson, G. Johansson, C. M. Wilson, and F. Nori, Phys. Rev. Lett. 103, 147003 (2009).

[24] See the Supplemental Material at http://link.aps.org/ supplemental/10.1103/PhysRevLett.124.140503 for details.

[25] W. C. Stewart, Appl. Phys. Lett. 12, 277 (1968).

[26] D. E. McCumber, J. Appl. Phys. 39, 2503 (1968).

[27] R. H. Koch, D. J. Van Harlingen, and J. Clarke, Phys. Rev. Lett. 45, 2132 (1980).

[28] L. Spietz, K. W. Lehnert, I. Siddiqi, and R. J. Schoelkopf, Science 300, 1929 (2003).

[29] L. Spietz, R. J. Schoelkopf, and P. Pari, Appl. Phys. Lett. 89, 183123 (2006).

[30] R. Simon, Phys. Rev. Lett. 84, 2726 (2000).

[31] G. Adesso and F. Illuminati, J. Phys. A 40, 7821 (2007).

[32] H. S. Ku, W. F. Kindel, F. Mallet, S. Glancy, K. D. Irwin, G. C. Hilton, L. R. Vale, and K. W. Lehnert, Phys. Rev. A 91, 042305 (2015).

[33] N. Treps and C. Fabre, Laser Phys. 15, 1 (2005).

[34] G. Giedke, M. M. Wolf, O. Krüger, R. F. Werner, and J. I. Cirac, Phys. Rev. Lett. 91, 107901 (2003).

[35] E. Flurin, N. Roch, F. Mallet, M. H. Devoret, and B. Huard, Phys. Rev. Lett. 109, 183901 (2012). 